\title{
QUELQUES OBSERVATIONS SUR LES MOTS TRONQUÉS DANS LE FRANÇAIS CONTEMPORAIN
}

\author{
Radka Fridrichová
}

\begin{abstract}
Nowadays, the economy of space and time is a major concern, reflected in people's lives, including the communication in their language evolving with the society which it reflects. It is perhaps this need to shorten that gave birth to the truncation - a phenomenon characteristic for modern French. The objective is to describe some features of this process, including the syllable structure, the relationship between terminal vowels and consonants, and the role of the apostrophe.

Keywords: clipping; shorten; syllable structure; final; graphic variants.

Résumé : De nos jours, l'économie de l'espace et du temps est une préoccupation importante, ce qui se répercute dans la vie des gens, dans leur communication et surtout dans leur langage qui évolue avec la société et la reflète. C'est peut-être la nécessité de faire court qui a donné naissance à la troncation - au phénomène caractéristique du français moderne. L'objectif de cet article est de décrire quelques traits de ce procédé, notamment la structure syllabique, le rapport entre les finales vocaliques et consonantiques et le rôle de l'apostrophe.
\end{abstract}

Mots clés : troncation ; abréger ; structure syllabique ; finale ; variantes graphiques.

\section{Introduction}

Dans cet article nous aimerions faire un point sur un procédé qui est très répandu dans le français contemporain - la troncation. Il puise dans les résultats de notre recherche concernant les mots tronqués effectuée pendant les études doctorales.

\section{Généralités, terminologie}

Ce procédé - la troncation/le mot tronqué, peut être appelé(e) un troncat. Certains linguistes $^{1}$ évitent cette appellation. Nous, dans le cadre de cet article, nous emploierons le mot troncat en tant que synonyme des mots tels que la troncation ou le mot tronqué. En outre, nous pouvons aussi utiliser les termes plus généraux : abrégé, abrègement, abréviation, raccourci, raccourcissement ou réduction pour toujours parler du procédé spécifique - la troncation.

\footnotetext{
Cf. notamment Gorcy qui souligne dans sa communication: «Certains linguistes appellent le mot tronqué un troncat : j'évoque ce terme pour ne pas l'employer par la suite. » (Gorcy $2000: 179)$
} 
Le Petit Robert 2009 nous apprend sur la troncation ceci : «Procédé d'abrègement d'un mot polysyllabique par suppression d'une ou plusieurs syllabes. Vélo est la troncation de vélocipède. ». Or, il nous renvoie aux expressions l'aphérèse et l'apocope. En les consultant, nous découvrons que l'apocope est formée par la «chute d'un phonème, d'une ou plusieurs syllabes à la fin d'un mot » et l'aphérèse, qui est son opposée, par la "chute d'un phonème ou d'un groupe de phonèmes au début d'un mot». Ces définitions sont convaincantes et largement acceptables.

Une seule chose qui est à objecter, c'est que Le Petit Robert ne mentionne pas les expressions populaires formées par la resuffixation en «-o ».

En outre, certains ouvrages classent, dans le processus de la troncation, la syncope, un procédé qui supprime un élément au centre du $\operatorname{mot}^{2}$ ( $c f$. notamment Pruvost - Sablayrolles 2003 : 114 ; Tournier $2009: 345$ ).

Rappelons que si la troncation est un phénomène lexical, l'abréviation est un phénomène uniquement graphique. L'abréviation est réduite à l'écrit (nous abrégeons le mot Monsieur en M., mais il est prononcé comme Monsieur, c'est-à-dire comme un mot entier), en revanche, la troncation peut être prononcée sous la forme de son abrègement (il est possible de dire la faculté ou, en utilisant la forme raccourcie, la fac). La dernière différence, c'est que les troncats ne prennent pas de point abréviatif ${ }^{3}$ et la plupart d'entre eux appartiennent à la langue familière, populaire et même argotique bien que leur présence dans la langue courante soit de plus en plus fréquente.

Les mots tronqués se forment généralement par la suppression de phonèmes initiaux ou finaux et ils se terminent fréquemment par une consonne. Ici, nous avons intentionnellement employé le mot phonème - et non syllabe - qui est également le terme utilisé par Le Petit Robert 2009 (PR09) et qui permet de mieux appréhender ce procédé. Notons que la coupure ne respecte pas toujours l'ordre syllabique, ce qui est le cas du mot scolarité qui s'abrège en scol (le mot est coupé au milieu de la syllabe) et non en sco ou scola. Selon Nicole et Jean Tournier, cette coupure est faite "sur une syllabe fermée, c'est-à-dire avec une consonne prononcée en finale, à partir de mots simples et composés ou d'expressions ». Ils expliquent qu' auparavant ce retranchement s'est produit «en une syllabe ouverte [...] plus souvent en deux syllabes (ciné) » (Tournier 2009 : 114).

Après notre propre analyse de périodiques et l'étude de la troncation dans ce domaine, nous sommes déjà en mesure de confirmer ou d'infirmer ces opinions. Les résultats sont présentés dans les lignes qui suivent.

\section{Choix du corpus}

Pour notre étude, nous avons choisi huit périodiques différents : Le Nouvel Observateur (NO), L'Express (EX), Cosmopolitan (CO), Marie France (MF), Elle (L), 20 minutes (20M), $\grave{A}$ nous Paris (ANP) et Les Inrockuptibles (INR). Il s'agit des magazines et des journaux dont les lecteurs ciblés sont très variés. Cela nous a permis d'avoir un échantillon et un lexique plus diversifiés et plus complexes.

Étant donné que nous avons choisi de soumettre à l'analyse le français contemporain, les unités sorties de notre recherche datent des années 2011 et 2012. Nous avons com-

\footnotetext{
2 Ce type d'abrègement est un peu spécifique car même si on supprime la partie médiane du mot à l'écrit, très souvent, ce changement n'apparaît pas à l'oral, contrairement aux abréviations graphiques.

3 Il ne s'agit pas d'une règle grammaticale mais d'une convention de typographie.
} 
mencé cette démarche au mois de janvier 2011 et ce processus s'est arrêté au mois de mars 2012. 200 journaux/magazines ont été soumis à notre analyse.

Notre corpus contient 590 mots abrégés. Nous avons exclu de ce corpus les nombres, les calques (agit-prop < agitation-propagande), les mots-valises tels que teknivals < techno + festivals, sitcom < situation comedy, biopic < biographical picture ou romcom < romantic comedy ainsi que les mots-valises formés à partir des noms propres Merkozy < Merkel + Sarkozy, etc. En revanche, nous avons classé dans notre corpus les mots d'origine étrangère, les noms propres, les troncats des mots composés et tous les mots qui se forment à l'aide de troncation en se servant, par exemples, du verlan, de la resuffixation ou de l'ellipse. Cependant, la simple ellipse (comme ne touche pas > pas touche) n'est pas prise en considération dans notre analyse.

\section{La structure syllabique}

\subsection{La structure syllabique des mots issus de troncation}

D'après Mitterand, le mode d'abréviation le plus usuel est celui qui ne conserve que les deux ou trois premières syllabes du mot de départ (Mitterand 1963 : 64).

Pareillement, les troncats dissyllabiques sont dans notre corpus prédominants (59\%), ce qui s'accorde d'ailleurs avec l'affirmation de Tournier citée ci-dessus. Ensuite, ce sont les unités monosyllabiques qui sont assez nombreuses (24\%) et les trisyllabiques comptant $15 \%$. En ce qui concerne l'analyse de Groud et Serna, nous avons reçu à peu près les mêmes résultats. Le corpus des deux linguistes comporte 120 monosyllabiques et 80 trisyllabiques, nos chiffres sont un peu plus élevés, 143 pour les mots qui gardent une seule syllabe et 86 qui en gardent trois. Ce fait peut être justifié par une augmentation des abréviations provenant de mots composés et de locutions. Quant aux troncats monosyllabiques, ils sont souvent d'origine anglaise (rappelons que nous avons inclus dans notre corpus les anglicismes) qui a une forte tendance au monosyllabisme. Enfin, nous nous apercevons que quelques exemples de troncats quadrisyllabiques (2\%) sont également présents dans notre analyse.

Le tableau $\mathrm{n}^{\circ} 1$ visualise cette analyse. Les juxtapositions des mots comme brico-déco ou déco-écolo ont été recomposées pour savoir comment ces mots ont été formés. Nous avons exclu de cette analyse le mot plouc, dont l'origine est inconnue, et les syncopes.

\begin{tabular}{|l|c|c|}
\hline \multicolumn{1}{|c|}{ Nombre de syllabes } & Nombre d'occurrences & $\begin{array}{c}\text { Nombre d'occurrences en } \\
\text { pourcentage }\end{array}$ \\
\hline Monosyllabiques & 143 & $24 \%$ \\
\hline Dissyllabiques & 348 & $59 \%$ \\
\hline Trisyllabiques & 86 & $15 \%$ \\
\hline Quadrisyllabiques & 9 & $2 \%$ \\
\hline Total & 586 & $100 \%$ \\
\hline
\end{tabular}

Tableau $n^{\circ} 1$ : Nombre de syllabes et d'occurrences des troncats 


\subsection{La structure syllabique des mots de départ}

Dans la même optique, nous avons analysé les mots de départ. Les résultats issus de cette analyse sont présentés dans le tableau $n^{\circ} 2$.

\begin{tabular}{|l|c|c|}
\hline \multicolumn{1}{|c|}{ Nombre de syllabes } & Nombre d'occurrences & $\begin{array}{c}\text { Nombre d'occurrences } \\
\text { en pourcentage }\end{array}$ \\
\hline Une & 12 & $2 \%$ \\
\hline Deux & 84 & $14 \%$ \\
\hline Trois & 191 & $32 \%$ \\
\hline Quatre & 203 & $35 \%$ \\
\hline Cinq & 76 & $13 \%$ \\
\hline Six & 16 & $3 \%$ \\
\hline Sept & 4 & $1 \%$ \\
\hline Total & 586 & $100 \%$ \\
\hline
\end{tabular}

Tableau $n^{\circ} 2$ : Nombre de syllabes et d'occurrences des mots de départ

Nous notons que les quadrisyllabiques (35\%) et les trisyllabiques (32\%) ont la position la plus forte. Ensuite, le tableau nous fait apparaître que les dissyllabiques (14 \%) et les pentasyllabiques (13\%) portent encore un pourcentage important. Le reste, c'est-à-dire les monosyllabiques, les hexasyllabiques et les heptasyllabiques sont plutôt marginaux. Les mots complets les plus courts sont par exemple femme, pote, for ou pauvre. En revanche, les mots les plus longs de notre corpus sont télécommunication, recapitalisation, etc.

\subsection{Abrégés ayant deux ou plusieurs formes}

Comme un même troncat peut contenir plusieurs significations, comprenons qu'il peut être issu de mots complets différents, à l'inverse, un même mot entier peut engendrer plusieurs abrégés dont le nombre de syllabes diffère. Ce fait est appelé par Groud et Serna une « surtroncation » (Groud - Serna 1993 : XVI).

Pour mieux illustrer ce qui vient d'être dit, citons quelques exemples découlant de notre répertoire :

$$
\begin{aligned}
& \text { anarchiste } \rightarrow \text { anarcho } \rightarrow \text { anar } \\
& \text { antifasciste } \rightarrow \text { antifacho } \rightarrow \text { antifa } \\
& \text { application } \rightarrow \text { appli } \rightarrow \text { app } \\
& \text { association } \rightarrow \text { assoce } \rightarrow \text { asso } \\
& \text { bricoleur } \rightarrow \text { birocolo } \rightarrow \text { bricol' }^{\prime} \\
& \text { célibataire } \rightarrow \text { célibate } \rightarrow \text { célib } \\
& \text { chocolat } \rightarrow \text { choco } \rightarrow \text { choc } \\
& \text { collaborateur } \rightarrow \text { collabo } \rightarrow \text { collab' } \\
& \text { documentaire } \rightarrow \text { docu } \rightarrow \text { doc } \\
& \text { populaire } \rightarrow \text { populo } \rightarrow \text { popu } \rightarrow \text { pop } \\
& \text { publicité } \rightarrow \text { publi } \rightarrow \text { pub } \\
& \text { toxicomane } \rightarrow \text { toxico } \rightarrow \text { tox }
\end{aligned}
$$

Il est à souligner que, parfois, une de ces formes raccourcies est attestée dans le dictionnaire. Une fois c' est la plus courte qu' on y trouve, d'autres fois, celle qui est plus longue. 
Par exemple, dans le PR09, nous discernons le mot assoce, mais non asso. À l'inverse, anar est attesté dans le PR09, mais pas anarcho. Voilà pourquoi il est difficile de dire, laquelle de ces formes est la plus ancienne et laquelle est la plus récente. Il arrive qu'aucun raccourci ne figure dans le dictionnaire et que ces deux formes puissent avoir une motivation de naissance différente. Nous sommes persuadée que c'est le cas du mot application, d'une part motivé par le français (appli), d'autre part influencé par l'abrègement anglais $(a p p)$. Ici encore, il est difficile de dire si le mot est d'abord abrégé en anglais et postérieurement adopté en français ou si d'abord, le mot est emprunté sous sa forme entière et ensuite raccourci. Toutefois, la réponse à ces questions, ne constitue pas l'objectif de ce travail.

Arrivée à ce point, il paraît indispensable de noter que cette «surtroncation » se fait au profit de la consonne à la fin du troncat, comme l'évoquent Groud et Serna (Groud Serna 1993 : XVI). Un exemple a gardé la voyelle finale : antifasciste $\rightarrow$ antifacho $\rightarrow$ antifa et un seul cas comprend un changement au profit de la finale vocalique : association $\rightarrow$ assoce $^{4} \rightarrow$ asso.

\subsection{La comparaison des structures syllabiques : du mot complet au raccourci par troncation}

En comparant les résultats précédents - comment les mots de départ sont abrégés et combien de syllabes sont enlevées, nous recevons le tableau n 3 . Les données (en chiffres) les plus considérables sont mises en gras et en italique.

\begin{tabular}{|l|c|c|c|c|c|c|c|}
\hline $\begin{array}{l}\text { Mots de départ (nombre } \\
\text { de syllabes) } \rightarrow \\
\text { Abrégés } \downarrow\end{array}$ & $\mathbf{1}$ & $\mathbf{2}$ & $\mathbf{3}$ & $\mathbf{4}$ & $\mathbf{5}$ & $\mathbf{6}$ & $\mathbf{7}$ \\
\hline Monosyllabiques & 6 & 55 & 52 & 22 & 8 & 0 & 0 \\
\hline Dissyllabiques & 6 & 29 & $\mathbf{1 2 0}$ & $\mathbf{1 4 3}$ & 43 & 6 & 1 \\
\hline Trisyllabiques & 0 & 0 & 18 & 37 & 20 & 9 & 2 \\
\hline Quadrisyllabiques & 0 & 0 & 1 & 1 & 5 & 1 & 1 \\
\hline Total & $\mathbf{1 2}$ & $\mathbf{8 4}$ & $\mathbf{1 9 1}$ & $\mathbf{2 0 3}$ & $\mathbf{7 6}$ & $\mathbf{1 6}$ & $\mathbf{4}$ \\
\hline
\end{tabular}

Tableau $\mathrm{n}^{\circ} 3$ : Comparaison des structures syllabiques

Ces entrées illustrent que les mots formés par troncation sont très souvent quadrisyllabiques et après ce procédé, ils deviennent dissyllabiques (143). Ce phénomène est suivi d'un nombre de 120 cas de trisyllabiques qui sont abrégés en dissyllabiques. Ensuite, il y a un écart entre les autres cas. Nous comptons environ 50 exemples de ces procédés : 2 syllabes $\rightarrow 1$ syllabe, 3 syllabes $\rightarrow 1$ syllabe et 5 syllabes $\rightarrow 2$ syllabes.

En observant le tableau, nous pouvons en tirer la conclusion suivante : les mots plus courts ayant deux ou trois syllabes au départ en suppriment une seule, tandis que les mots de départ plus longs en enlèvent deux (les quadrisyllabiques) ou trois (les pentasyllabiques ou les hexasyllabiques).

Présentons maintenant, sous forme d'un tableau (tableau $n^{\circ} 4$ ), quelques exemples de procédés de raccourcissement au niveau syllabique.

\footnotetext{
4 Le «e » muet n'est pas ici considéré comme une finale vocalique.
} 
Radka Fridrichová

\begin{tabular}{|c|c|c|}
\hline $\begin{array}{l}\text { Nombre de syllabes } \\
\text { (mot de départ }>\text { mot } \\
\text { issu de troncation) }\end{array}$ & $\begin{array}{l}\text { Mot complet }>\text { mot } \\
\text { issu de troncation }\end{array}$ & Exemple en contexte \\
\hline 7 syllabes $>2$ syllabes & $\begin{array}{l}\text { recapitalisation }> \\
\text { recap }\end{array}$ & «Recap à contrecour » (NO, n² 2450, p. 30) \\
\hline 7 syllabes $>3$ syllabes & $\begin{array}{l}\text { télécommunication > } \\
\text { télécom }\end{array}$ & $\begin{array}{l}\text { «Télécoms, ton univers impitoyable! » }\left(20 \mathrm{M}, \mathrm{n}^{\circ} 2101 \text {, }\right. \\
\text { p. 18) }\end{array}$ \\
\hline 7 syllabes $>4$ syllabes & $\begin{array}{l}\text { multikulturalismus }> \\
\text { multikulti }\end{array}$ & $\begin{array}{l}\text { "Après des mois de polémiques et de discours déclinistes } \\
\text { (initiés par la publication du pamphlet xénophobe de } \\
\text { Thilo Sarrazin), l'Allemagne redécouvre les joies du } \\
\text { "multikulti " (multiculturalisme) avec le film Almanya } \\
\left.\text { - Wilkommen in Deutschland. " (INR, } \mathrm{n}^{\circ} 813, \mathrm{p} .11\right)\end{array}$ \\
\hline 6 syllabes $>2$ syllabes & kinésithérapie > kiné & $\begin{array}{l}\text { "Prudence avec la kiné, l'ostéopathie et la chiropratique } \\
\text { en phase aiguë." ( }\left(\mathrm{MF}, \mathrm{n}^{\circ} 202, \mathrm{p} .119\right)\end{array}$ \\
\hline 6 syllabes $>3$ syllabes & $\begin{array}{l}\text { désintoxication }> \\
\text { désintox }\end{array}$ & $\begin{array}{l}\text { «Info ou désintox? Fort de sa saison dans l'enfer des } \\
\text { substances toxiques, Jean-Luc Delarue revient pour } \\
\left.\text { rabibocher les familles désunies. " (INR, } \mathrm{n}^{\circ} 822, \mathrm{p} .117\right)\end{array}$ \\
\hline 6 syllabes $>4$ syllabes & $\begin{array}{l}\text { cyberréactionnaire > } \\
\text { cyberréac }\end{array}$ & $\begin{array}{l}\text { "- le cancer de l'internet pour certains cyberréacs -, " } \\
\left(\mathrm{INR}, \mathrm{n}^{\circ} 837, \mathrm{p} .118\right)\end{array}$ \\
\hline 5 syllabes $>1$ syllabe & $\begin{array}{l}\text { communication }> \\
\text { com }\end{array}$ & $\begin{array}{l}\text { "L'idée: entrer dans l'univers des pros de la com, et ren- } \\
\text { contrer ses acteurs. " (ANP, } \mathrm{n}^{\circ} 517, \text { p. 6) }\end{array}$ \\
\hline 5 syllabes $>2$ syllabes & $\begin{array}{l}\text { manipulation > } \\
\text { manip }\end{array}$ & $\begin{array}{l}\text { "Et si c'était une manip, après tout? Et de la part de } \\
\left.\text { qui? " (INR, } \mathrm{n}^{\circ} 814, \mathrm{p} .10\right)\end{array}$ \\
\hline 5 syllabes $>3$ syllabes & aristocratique $>$ aristo & «Allure aristo et pop noble » (INR, $\mathrm{n}^{\circ} 828$, p. 92) \\
\hline 5 syllabes $>4$ syllabes & $\begin{array}{l}\text { autopromotion }> \\
\text { autopromo }\end{array}$ & $\begin{array}{l}\text { "Cet hiver s'annonce bien, voire beau comme } \\
\text { l'autopromo du moment pour une grosse chaîne de télé: } \\
\text { côté couleurs qu'il faut, il y a le rouge... " (ANP, n } 539 \text {, } \\
\text { p. 20) }\end{array}$ \\
\hline 4 syllabes $>1$ syllabe & $\begin{array}{l}\text { programmation }> \\
\text { prog }\end{array}$ & $\begin{array}{l}\text { «On ne pouvait donc qu'attendre moquette et mobilier } \\
\left.\text { d'époque pour une prog pointue. » (INR, } \mathrm{n}^{\circ} 814, \mathrm{p} . \mathrm{XV}\right)\end{array}$ \\
\hline 4 syllabes $>2$ syllabes & décoration > déco & $\begin{array}{l}\text { "Désormais, ils s'essaient à quelques éléments de déco. " } \\
\left(\mathrm{NO}, \mathrm{n}^{\circ} 2426, \mathrm{p} .132\right)\end{array}$ \\
\hline 4 syllabes $>3$ syllabes & apéritif > apéro & $\begin{array}{l}\text { "Mon maître de stage m'a demandé de nous servir un } \\
\text { pastis pour l'apéro. " }\left(\mathrm{NO}, \mathrm{n}^{\circ} 2465, \mathrm{p} .75\right)\end{array}$ \\
\hline 4 syllabes $>4$ syllabes & $\begin{array}{l}\text { antifasciste }> \\
\text { antifacho }\end{array}$ & $\begin{array}{l}\text { "Il y a chez eux une impossibilité matricielle à pouvoir } \\
\text { faire de la politique car un anarchiste brésilien, un liber- } \\
\text { tin américain ou un antifacho allemand } n^{\prime} \text { ont rien de } \\
\left.\text { commun politiquement parlant. » (INR, } \mathrm{n}^{\circ} 845, \mathrm{p} .34\right)\end{array}$ \\
\hline 3 syllabes $>1$ syllabe & production $>$ prod & $\begin{array}{l}\text { "A l'époque, Sébastian, } 20 \text { ans, n'a réalisé qu'une poi- } \\
\text { gnée de prods pour les rappeurs de La Cliqua et quelques } \\
\left.\text { autres «trucs hybrides»." (INR, } n^{\circ} 812, \text { p. } 48\right)\end{array}$ \\
\hline 3 syllabes $>2$ syllabes & bigophone $>$ bigo & «Buzz: accros du bigo » $\left(\mathrm{CO}, \mathrm{n}^{\circ} 461, \mathrm{p} .98\right)$ \\
\hline 3 syllabes $>3$ syllabes & islamiste $>$ islamo & $\begin{array}{l}\text { «Lui et son adjoint, Jean-François Ricard, se réservent les } \\
\text { «islamo », Gilbert Thiel hérite des Corses, Laurence Le } \\
\left.\text { Vert des Basques. » (NNO, }{ }^{\circ} 2467, \text { p. } 81\right)\end{array}$ \\
\hline
\end{tabular}


Quelques observations sur les mots tronqués dans le français contemporain

\begin{tabular}{|c|c|c|}
\hline 3 syllabes $>4$ syllabes & oligarque $>$ oligogo & $\begin{array}{l}\text { "Il pourrait se qualifier lui-même d' "oligogo ", un } \\
\text { type qui croyait dur comme fer à l'élite à la française, } \\
\text { à ses mythes fondateurs, et qui a été trompé sur la } \\
\text { marchandise. " }\left(\mathrm{NO}, \mathrm{n}^{\circ} 2427, \mathrm{p} .76\right)\end{array}$ \\
\hline 2 syllabes $>1$ syllabe & problème > blème & $\begin{array}{l}\text { "On emploie la métaphore (les «airbags » pour les seins), } \\
\text { le verlan monosyllabique (" einb " pour bien), l'apocope } \\
\text { (" basks " pour baskets), l'aphérèse (" blème " pour } \\
\left.\text { problème), etc. " (MF, } \mathrm{n}^{\circ} 190, \text { p. } 93\right)\end{array}$ \\
\hline 2 syllabes $>2$ syllabes & gauchiste $>$ gaucho & $\begin{array}{l}\text { «Il y a tellement de jolis petits romans, pleins de bonne foi } \\
\text { sociétale avec un côté gaucho propret. » (NO, } \mathrm{n}^{\circ} 2463 \text {, } \\
\text { p. } 96)\end{array}$ \\
\hline 1 syllabe $>1$ syllabe & flic $>$ keuf & $\begin{array}{l}\text { «Si «keuf» et « meuf» font depuis longtemps partie du } \\
\text { paysage, "geek» ou « sex-toy » viennent d'apparaître. » } \\
\left(\mathrm{MF}, \mathrm{n}^{\circ} 190, \mathrm{p} .93\right)\end{array}$ \\
\hline 1 syllabe $>2$ syllabes & pote $>$ poto & $\begin{array}{l}\text { "Pour le } 31 \text { décembre, il a convié trois cents potos rigolos } \\
\text { à Saint-Barth pour une petite teuf sans prétention, avec } \\
\text { un petit groupe coolos pour animer baloche." (INR, } \\
n^{\circ} 841, \text { p. } 67 \text { ) }\end{array}$ \\
\hline
\end{tabular}

Tableau $\mathrm{n}^{\circ} 4$ : Comparaison des structures syllabiques (mot de départ vs mot final)

Nous pouvons relever du tableau plusieurs éléments intéressants. Premièrement, c'est le nombre de syllabes qui n'a pas changé en comparant le mot de départ et le mot final. Cela peut être dû au fait que le mot tronqué est a posteriori suivi de resuffixation. Cela affecte surtout les mots trisyllabiques (islamo < islamiste, onano < onaniste, populo < populaire, etc.). Même si le nombre de syllabes ne change pas, la terminaison («-o ») employée après troncation est plus courte que celle du mot d'origine, il s'agit des suffixes tels que : «-iste», «-ique » ou «-ment». Ici, nous parlons toujours de l'économie des mots. Parfois, le suffixe est aussi long que celui du mot de départ (sympathique > sympatoche). Les redoublements hypocoristiques entraînent également ce non-changement de nombre de syllabes, le plus souvent, ils influencent les dissyllabiques (Lolo < Laurent, Mimi < Rémi, Jojo < Johnny/Jordan). Par contre, dans ces deux derniers cas, il n'y a pas de question d'économie de temps ou d'espace par les mots finaux. Vu qu'il ne s'agit pas de la simple troncation, l'objectif du procédé suivant (la resuffixation ou le redoublement) est d'obtenir une connotation plus familière du mot et la troncation sert alors d'intermédiaire.

Deuxièmement, nous remarquons que le nombre de syllabes du mot final est plus élevé que celui du mot de départ. Nous avons noté un seul exemple - parlons plutôt d'une exception, pour le lexème trisyllabique qui est devenu quadrisyllabique (oligarque $>$ oligogo). De nouveau, c'est le redoublement de la dernière syllabe qui a créé cette augmentation de syllabes. Pour les monosyllabiques qui sont devenus dissyllabiques, nous comptons déjà six occurrences (Jacques $>$ Jako, pote $>$ poto, tante $>$ tata). L'accroissement de syllabes se déroule par redoublement ou par resuffixation qui suivent la troncation.

Enfin, il est curieux de remarquer que le mot multikulti est une apocope du mot allemand multikulturalismus qui est heptasyllabique, alors que sa version française n' est que hexasyllabique - multiculturalisme. Dans notre comptage, nous avons gardé son origine et nous l'avons incorporé parmi les heptasyllabiques. 


\section{Point de troncature}

Le point de troncature est l'endroit où la troncation s'est produite. Cette dernière ne respecte pas toujours la coupe syllabique, c'est-à-dire la construction du mot de départ. Cette irrégularité ne représente pas une exception, elle touche $37 \%$ des troncats. Les $11 \%$ de ce nombre constituent les mots tronqués à partir de l'intérieur d'une syllabe suivis de resuffixation ( $c f$. tableau $\mathrm{n}^{\circ} 5$ ).

\begin{tabular}{|l|c|c|}
\hline Point de troncature & Pourcentage & Exemple \\
\hline Coupe syllabique & $63 \%$ & techno-crate \\
\hline À l'intérieur d'une syllabe & $26 \%$ & mag-azine \\
\hline À l'intérieur d'une syllabe + resuffixation & $11 \%$ & popul-o > popul-aire \\
\hline
\end{tabular}

Tableau $\mathrm{n}^{\circ} 5$ : Point de troncature (corpus)

Maintenant, faisons remarquer quelques spécificités concernant le point de troncature.

Il se peut que deux morphèmes existent séparément : kilo-gramme. Le plus souvent, ce sont les mots provenant de la dérivation d'un nom d'origine grecque ou latine. La troncation se produit entre ces deux morphèmes.

Parfois, cette coupe n'est pas respectée, c'est le cas du mot écologie ou de son dérivé écologique, issus du grec : «oikos » et «logos ». Si la troncation suivait le modèle grec, la coupe devrait se faire entre éco et logique. Mais elle ne le suit pas et le mot se coupe comme ceci : écolo-gique.

À l'inverse, le morphème de base d'un mot ne doit pas respecter la coupe syllabique, tandis que la troncation la soigne. Prenons l'exemple du mot décorer, dont la racine est « décor- », la coupe est alors "irrégulière » et à partir de cette racine, nous formons d'autres mots en ajoutant des suffixes, "-atif », "-ation ", "-ateur », etc. Au moment où nous raccourcissons les mots dérivés, nous obtenons le troncat déco qui respecte la coupe syllabique.

Enfin, les deux - le morphème de base + suffixe et la troncation - ne doivent pas tenir compte de la coupe syllabique : la climatisation se coupe en cli-ma-ti-sa-tion, le morphème de base est climatis-ation et la troncation étant : clim-atisation.

L'objectif essentiel de la troncation est de faire court, parfois, d'abréger le mot le plus possible, ce qui peut poser des problèmes de compréhension. Si le locuteur ne connaît pas le mot de départ correspondant à l'abrégé, il risque des confusions de significations.

\section{La finale}

Nous avons déjà décrit les structures syllabiques des troncats que nous avons repérés dans les périodiques français. Ci-après, nous allons prêter attention à la distinction entre la finale vocalique et la finale consonantique.

Cette finale est étudiée du point de vue de la phonétique, c'est la raison pour laquelle le « e » muet précédé d'une consonne n'est pas ici considéré comme une finale vocalique. Nous n'avons pas tenu compte des mots nés par aphérèse et par syncope parce que leur finale reste sans changement et les mots juxtaposés ont été recomposés. Les mots ayant plusieurs signifiés ou signifiants ont été comptés pour chacun de ces cas. 
Lorsque nous regardons la répartition des finales (tableau $n^{\circ} 6$ ), nous constatons que les finales vocaliques (65\%) ont l'avantage sur les finales consonantiques (35\%). Ces dernières proviennent, dans la plupart des cas, des troncats monosyllabiques (prof $<$ professeur, prod < production, sup < supérieur/supplémentaire), com < communication, bad < badminton, mag < magazine, cam < caméra, etc.). Les finales en « o » sont prédominantes (36 \%). Les finales vocaliques resuffixées en «-o » représentent $9 \%$ et les autres vocaliques $20 \%$.

\begin{tabular}{|l|c|c|c|}
\hline Finale & Nombre & Pourcentage & Exemple \\
\hline Finales vocaliques en « o » & 211 & $36 \%$ & collabo \\
\hline Finales vocaliques resuffixées en «-o » & 49 & $9 \%$ & intello \\
\hline Les autres finales vocaliques & 116 & $20 \%$ & Répu \\
\hline Finales consonantiques & 201 & $35 \%$ & compil \\
\hline
\end{tabular}

Tableau $n^{\circ} 6$ : Répartition des finales

En consultant d'autres corpus qui traitent les troncats, ceux de Groud et Serna (1996), d'Antoine (1993), de George (1980), nous constatons que le nôtre se rapproche le plus des résultats d'Antoine. Il a repéré $67 \%$ de finales vocaliques contre $33 \%$ de finales consonantiques. Le corpus des autres linguistes se divisent à peu près en 2 parties équivalentes. Nous pensons que ce fait peut s'expliquer par une présence plus élevée de la finale vocalique en [o] dans notre corpus, il compte $70 \%$ de toutes les finales vocaliques, tandis que dans le corpus de Groud et Serna, ils en comptent $60 \%$. Notre analyse correspond, de nouveau, plus aux données de George et d'Antoine (Groud - Serna 1993 : XIV). Groud et Serna ont exclu de leur corpus les vocables déjà attestés dans les dictionnaires tels que aristo, mélo, chromo, etc., ce qui peut justifier la baisse des vocaliques en [o].

Lorsque nous étudions plus en détail la répartition des finales vocaliques ( $c f$. tableau $\mathrm{n}^{\circ} 7$ ), nous nous apercevons que les finales en « $\mathrm{O} »-$ sans distinguer les substitutions de voyelle finale par «-o », constituent $70 \%$. Les apocopés en «-i » constituent $13 \%$, les finales en «-a » et «-é » ont encore un pourcentage considérable de $8 \%$ et $5 \%$. Le reste est plutôt marginal et exceptionnel. De ce fait, nous pouvons déduire que la terminaison « $\mathrm{O}$ » est prise soit pour une finale des apocopés soit pour un suffixe substitutionnel et occupe une place incontournable ( $45 \%$ de l'ensemble du corpus) dans la formation des mots par troncation.

\begin{tabular}{|c|c|c|c|}
\hline Finale & Nombre & Pourcentage & Exemple \\
\hline [o] & 260 & $70 \%$ & bio $[$ bjo] \\
\hline [i] & 51 & $14 \%$ & ampli [ãpli] \\
\hline [a] & 29 & $8 \%$ & sympa [s⿱乛⿻上丨a] \\
\hline [e] & 19 & $5 \%$ & synthé [sẽte] \\
\hline [y] & 12 & $3 \%$ & $a c t u$ [akty] \\
\hline$[\tilde{\jmath}]$ & 3 & $0,8 \%$ & fonfon [โวิ์ว̃] \\
\hline$[\varnothing]$ & 2 & $0,5 \%$ & pneu [pnø] \\
\hline
\end{tabular}

Tableau $n^{\circ} 7$ : Répartition des finales vocaliques 
Concernant les finales consonantiques, $c^{\prime}$ est la consonne [k] qui a un taux de fréquence plus élevé que les autres consonnes. La consonne $[\mathrm{k}]$ correspond phonétiquement à plusieurs lettres - en prenant en compte les règles de prononciation, par exemple : dans le mot tech [tck], nous écrivons le digramme "ch » [S] à la fin, mais nous le prononçons comme un [k]. Pareillement, la lettre « $\mathrm{c}$ » se prononce sous certaines conditions comme un [k] : renc [Rãk] comme le groupe de lettres «-que » : zique [zik]. Cela peut justifier la plus grande fréquence de la consonne $[\mathrm{k}]$. Le tableau $\mathrm{n}^{\circ} 8$ laisse voir la répartition totale des finales consonantiques.

\begin{tabular}{|c|c|c|c|}
\hline Finale & Nombre & Pourcentage & Exemple \\
\hline$[\mathrm{k}]$ & 35 & $17 \%$ & coloc [kolok] \\
\hline [p] & 23 & $11 \%$ & $a p p$ [ap] \\
\hline [s] & 23 & $11 \%$ & assoce [asos] \\
\hline$[\mathrm{t}]$ & 20 & $10 \%$ & basket [basket] \\
\hline$[\mathrm{b}]$ & 18 & $9 \%$ & célib [selib] \\
\hline [f] & 12 & $6 \%$ & manif [manif] \\
\hline$[\mathrm{m}]$ & 13 & $6 \%$ & clim [klim] \\
\hline [S] & 11 & $5,5 \%$ & kalach [kalaf] \\
\hline [R] & 10 & $5 \%$ & roler [RolœR] \\
\hline [v] & 8 & $4 \%$ & couv'/couve [kuv] \\
\hline [d] & 6 & $3 \%$ & prod [prod] \\
\hline [3] & 6 & $3 \%$ & bourge [buR3] \\
\hline [1] & 6 & $3 \%$ & stal [stal] \\
\hline [n] & 4 & $2 \%$ & fan [fan] \\
\hline [z] & 4 & $2 \%$ & occase [okaz] \\
\hline [g] & 2 & $1 \%$ & $m a g[\mathrm{mag}]$ \\
\hline
\end{tabular}

Tableau $\mathrm{n}^{\circ} 8$ : Répartition des finales consonantiques

Relevons du tableau deux éléments des plus curieux. Premièrement, c'est le phonème [S], dont les 11 cas sont en général les adjectifs qui se terminent par un suffixe en «-oche »:

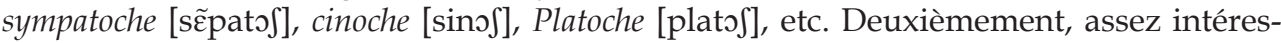
sant est le cas des mots qui se terminent par la lettre « $\mathrm{n}$ » précédée d'une voyelle. Quand ils sont d'origine française, il y a une nasale à la fin : colon [kolõ], néocon [neokõ]. Dès qu'ils viennent de l'anglais, le « $\mathrm{n}$ » est prononcé et la prononciation française ne s'adapte pas et la nasale non plus : fan [fan], skin [skin].

Enfin, nous accentuons qu'aucune consonne finale ne présente, dans l'ensemble, de résultats fréquents aussi importants que la finale vocalique en « $\mathrm{O} »$. 


\section{La graphie}

La question de la graphie comporte deux éléments des plus importants. Ce sont les variantes graphiques qui changent lors des modifications phonétiques et l'apostrophe dont l'emploi n'est toujours pas régulier.

\subsection{Les variantes}

Comme nous l'avons déjà abordé, les modifications phonétiques peuvent entraîner des modifications dans l'écriture d'un troncat, c'est-à-dire des modifications graphiques. Cependant, la prononciation ne change pas.

Voici quelques exemples issus de notre corpus :

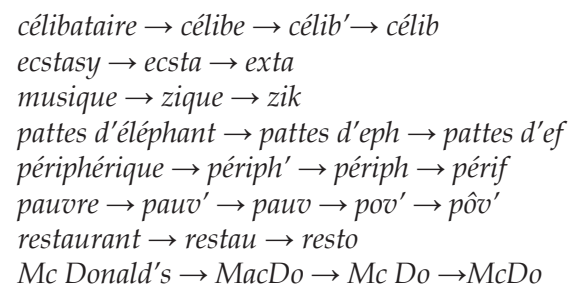

Nous nous apercevons que pour le mot pauvre nous avons une vraie variété de graphies, même sans ou avec apostrophe.

\subsection{Question de l'apostrophe}

Lacroux affirme que «l'apostrophe est une timidité» (Lacroux 2007 : 307). Au contraire, Groud et Serna prétendent que l'emploi de l'apostrophe est de plus en plus fréquent (Groud - Serna 1993 : XVI). Leur corpus contient 107 abrégés suivis de l'apostrophe, le nôtre en compte à peu près la moitié $(50$ cas). Cela reflète peut-être notre moins importante présence des finales consonantiques dans l'ensemble du corpus qui sont plus souvent, à la différence des finales vocaliques, accompagnées de l'apostrophe.

En outre, les apostrophes servent de marque du pluriel pour les adjectifs et les substantifs.

«[...] rien à voir avec les personnages ringards et réac' qu'il a crée à la fin des années 70. » (INR, $\left.\mathrm{n}^{\circ} 848, \mathrm{p} .83\right)$;

«Exit les concours de beauté, vive les concours de style! Ces nouvelles compét' font un tabac auprès des jeunes. » (NO, $\left.\mathrm{n}^{\circ} 2428, \mathrm{p} .124\right)$.

Mais cet emploi de l'apostrophe dans notre corpus a plutôt été exceptionnel. Dans la plupart des cas, l'apostrophe a été employée au singulier après une consonne et dans trois cas après une voyelle (coloration $>$ colo', gin tonic $>$ gin to', for $>f o^{\prime}$ ), ce qui prouve l'affirmation de Lacroux selon laquelle les apostrophes ne sont quasiment jamais utilisées après une voyelle (Lacroux 2007 : 307).

«Bon en 2012, qui compte être malade et quand ??? Mois je prends Avril, comme d'hab'! (MF, $\mathrm{n}^{\circ} 200$, p. 190)

«Le premier single En transe... ylvanie (rapport à la Transylvanie, tsé) est d'ores et déjà prometteur ( En Transylvanie, un trans' se maquille ) mais pas plus que le nouveau show de Dove Attia intitulé 1789. » (INR, $\mathrm{n}^{\circ} 832$, p. 28) ; 
«Grandes, petites, rondes, filiformes, brunes, blondes, rousses, ces < reines du style > n'ont rien des Kate Moss et consorts mais sont shootées comme des mannequins (la couv' est même signée par Karl Lagerfeld). » (NO, $\mathrm{n}^{\circ} 2428$, p. 124).

L'apparition de l'apostrophe est bien fréquente dans les abrégés de noms propres.

"Le Cav" au sommet du monde. A 26 ans, Mark Cavendish est devenu champion du monde sur route hier à Copenhague. » (20M, n² 2111, p. 15);

«Mais quand Jean-Mi' la voit, c'est le drame. » (ANP, $\mathrm{n}^{\circ}$ 530, p. 36) ;

«Bardot, c'est à Saint-Trop', mais la Brigitte c'est à Calvi. » (L, nº 3418, p. 38).

Ainsi, il y a des mots qui engendrent les deux formes - sans apostrophe et avec apostrophe (périphérique > périph/périph', récupération > récup/récup', communication > com/

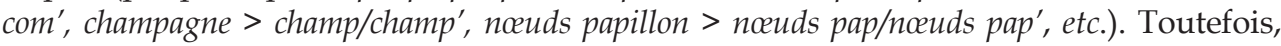
l'unique forme avec apostrophe a été assez abondante (gravement $>$ grav', asiatique $>$ asiat', bricoleur $>$ bricol', collaboration $>$ collab' ou maternité $>$ mat').

L'apostrophe est en général placée à la fin du mot pour marquer que le mot a été apocopé. Il peut se retrouver aussi au début du mot où il indique l'autre procédé, celui de l'aphérèse. Comme l'aphérèse est plus rare que l'apocope, l'apostrophe n'y apparaît que deux fois ('tit < petit,'Jour < Bonjour).

L'essentiel est de noter que l'apostrophe nous informe sur le procédé de raccourcissement. Elle signale également la suppression du « -e » final d'un troncat et en même temps, la conservation de sa prononciation.

"Car la réussite d'un centreur dépend avant tout de celle de son buteur. Dès qu'il donne un ballon,

l'ailier cherche la < passe déc' > ». (20M, n²135, p. 15)

L'apostrophe nous signale la prononciation du décisive [desiziv] $\rightarrow$ déc' [des]. Pareillement,

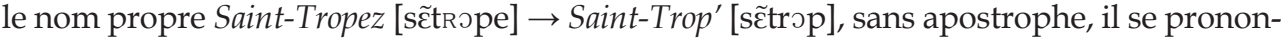
cerait Saint-Trop [sc̃tro]. Malheureusement, nous ne pouvons pas parler d'une règle car l'apostrophe, qui note la conservation de prononciation, ne s'emploie pas à chaque fois. Pour l'illustrer, nous citions l'abrégé du champagne $\rightarrow$ champ/champ'. Nous avons repéré dans notre corpus les deux écritures, selon « la règle » que nous avons formulée ci-dessus, le mot champ se prononcerait [ $\left.\int \tilde{\mathrm{a}}\right]$ et champ' deviendrait [ $\left.\int \tilde{\mathrm{a}} \mathrm{p}\right]$. Mais cette affirmation serait fausse parce que le « $\mathrm{p}$ » est prononcé dans les deux cas, ce qui est aussi noté dans la transcription phonétique du $P R$ ou d'autres dictionnaires. De plus, l'abrégé peut être confondu avec le mot champ = terrain, espace, terre propre à la culture.

Il découle de ce court parcours qu'une réponse exacte à la question de l'apostrophe ne peut pas être donnée. L'apostrophe est justifiée, p. ex. pour distinguer un abrégé d'un mot entier qui possède le même signifiant ou pour garder la prononciation d'une finale. Néanmoins, cela n'est pas toujours respecté et tout dépend du libre choix du créateur du texte.

\section{Conclusion}

Les mots formés par troncation - le phénomène étudié dans cet article, pénètrent dans la langue française contemporaine de manière de plus en plus évidente, ils sont généralement connus et souvent employés, surtout par les jeunes. Le besoin de faire court a peutêtre créé la troncation. Nous osons remarquer que c'est également le besoin de ludisme qui était présent à la naissance des troncations. Elles servent de moyen d'attirer l'attention du lecteur de textes journalistiques au premier coup d'œil. 
Quelques observations sur les mots tronqués dans le français contemporain

En s'appuyant sur ces nouvelles créations langagières, les journalistes s'efforcent d'être plus proches du parler des locuteurs ou des lecteurs potentiels et de les persuader de les lire car ils correspondent aux nouvelles tendances de la langue française. Ils s'adressent à nous dans notre langage.

L'actualisation des écrits correspondent aux tendances de la langue parlée et à l'inverse sous l'influence de la presse et des médias, le discours de la société adopte les structures utilisées par ce type de communication.

Pour cette raison, l'évolution de ce phénomène recherché mérite d'être analysé constamment et il faut s'apercevoir de ces changements au cours du temps.

\title{
Bibliographie
}

ARrivé, Michel et al. (1986), La grammaire d'aujourd'hui: guide alphabétique de linguistique française, Paris : Flammarion.

BLOIS, Jacques - BAR, Marc, (1975), Notre langue française, Paris : Didier.

DAuZET, Albert et al. (1971), Nouveau dictionnaire étymologique et historique, Paris: Larousse.

DuBoIs, Jean (1965), Grammaire structurelle du français : nom et pronom, Paris : Larousse.

GAUdIn, François - GuesPin, Louis (2000), Initiation à la lexicologie française, Bruxelles : Duculot.

Gorcy, Gérard (2000), « La Mode de l'abréviation et de la troncation verbale en français contemporain ", Mémoires de l'Académie de Stanislas 15, 179-189.

GoudailLer, Jean-Pierre (2001), Comment tu tchatches, Paris : Maisonneuve et Larose.

Grevisse, Maurice (1993), Le bon usage, Paris : Duculot (13e éd. refondue par André Goose).

GROUD, Claudette - SERNA, Nicole (1996), De abdom à zoo, Regards sur la troncation en français contemporain, Paris : Didier Érudition.

Lacroux, Jean-Pierre (2007), Orthotypographie, Custines : Hélio Service. Disponible sur http:/ / creativecommons.org/licenses/by-nc-nd/2.0/fr/, consultéle 13/09/2011.

Le Petit Robert de la langue française 2009 (2009), Paris : Dictionnaires Le Robert.

LeHMANN, Alise - MARTIN-Berthet, Françoise (2003), Introduction à la lexicologie, Paris : Armand Colin.

Martinet, André (1979), Grammaire fonctionnelle du français, Paris : Didier.

Mitterand, Henri (1963), Les mots français, Paris : PUF.

Mortureux, Marie-Françoise (2008), La lexicologie entre langues et discours, Paris : Armand Colin.

Niklas-Salminen, Aïno (1997), La lexicologie, Paris : Armand Colin.

Pruvost, Jean - Sablayrolles, Jean-François (2003), Les néologismes, Que sais-je?, Paris : PUF.

TOURnIER, Nicole - TourniER, Jean (2009), Dictionnaire de la lexicologie française, Paris : Ellipses.

\author{
Radka Fridrichová \\ Fakulta pedagogická \\ Západočeská univerzita v Plzni \\ Veleslavínova 42 \\ 30614 Plzeň \\ République tchèque \\ RFridrichova@seznam.cz
}

